\title{
Effects of an oral allosteric AKT inhibitor (MK-2206) on human nasopharyngeal cancer in vitro and in vivo
}

This article was published in the following Dove Press journal:

Drug Design, Development and Therapy

10 October 2014

Number of times this article has been viewed

\author{
Yuan-Yuan Zhaol,* \\ Ying $\operatorname{Tian}^{1}$,* \\ Jing Zhang ${ }^{2, *}$ \\ Fei $X u^{\prime}$ \\ Yun-Peng Yang' \\ Yan Huang' \\ Hong-Yun Zhao ${ }^{3}$ \\ Jian-Wei Zhang ${ }^{4}$ \\ Cong Xue' \\ Michael H Lam ${ }^{5}$ \\ Li Yan ${ }^{5}$ \\ Zhi-Huang $\mathrm{Hu}^{\prime}$ \\ Xiao-Xiao Dinglin ${ }^{6}$ \\ Li Zhang ${ }^{1,3}$
}

'Department of Medical Oncology, Sun Yat-Sen University Cancer Center, State Key Laboratory of Oncology in South China, and Collaborative Innovation Center for Cancer Medicine, Guangzhou, People's Republic of China; ${ }^{2}$ Department of Medical Oncology, the First Affiliated Hospital of Guang Zhou Traditional Chinese Medicine University, Guangzhou, People's Republic of China; ${ }^{3}$ National Anti-Cancer Drug Research Centre, Sun Yat-Sen University Cancer Center; State Key Laboratory of Oncology in South China, and Collaborative Innovation Center for Cancer Medicine, Guangzhou, People's Republic of China;

${ }^{4}$ The Six Affiliated Hospital of Sun Yat-Sen

University, Guangzhou, People's Republic of China; ${ }^{5}$ Merck and Co Inc, North Wales, PA USA; 'Sun Yat-Sen Memorial Hospital, Guangzhou, People's Republic of China

*These authors contributed equally to this work

Correspondence: Li Zhang

Department of Medical Oncology, Sun

Yat-Sen University Cancer Centre, State

Key Laboratory of Oncology in South China,

65I Dong Feng Road East, Guangzhou 510060,

People's Republic of China

Tel +86 2087342288

Fax +86 2087343365

Email zhangli6@mail.sysu.edu.cn
Aim: Protein kinase B (AKT) signaling frequently is deregulated in human cancers and plays an important role in nasopharyngeal carcinoma (NPC). This preclinical study investigated the effect of MK-2206, a potent allosteric AKT inhibitor, on human NPC cells in vitro and in vivo.

Methods: The effect of MK-2206 on the growth and proliferation of CNE-1, CNE-2, HONE-1, and SUNE-1 cells was assessed by Cell Counting Kit 8 and colony formation assay. Flow cytometry was performed to analyze cell cycle and apoptosis. The effects of MK-2206 on the AKT pathway were analyzed by Western blotting. Autophagy induction was evaluated via electron microscopy and Western blot. To test the effects of MK-2206 in vivo, CNE-2 cells were subcutaneously implanted into nude mice. Tumor-bearing mice were treated orally with MK-2206 or placebo. Tumors were harvested for immunohistochemical analysis.

Results: In vitro, MK-2206 inhibited the four NPC cell line growths and reduced the sizes of the colonies in a dose-dependent manner. At 72 and 96 hours, the half maximal inhibitory concentration $\left(\mathrm{IC}_{50}\right.$ ) values of MK-2206 in CNE-1, CNE-2, and HONE-1 cell lines were 3-5 $\mu \mathrm{M}$, whereas in SUNE-1, $\mathrm{IC}_{50}$ was less than $1 \mu \mathrm{M}$, and MK-2206 induced cell cycle arrest at the G1 phase. However, our study found no evidence of apoptosis. MK-2206 induced autophagy in NPC cells, as evidenced by electron microscopy and Western blot, and inhibited the growth of tumors that were subcutaneously implanted in mice. Inhibition of downstream phosphorylation through the PRAS40 and S6 pathways seems to be the main mechanism for the MK-2206-induced growth inhibition.

Conclusion: Our preclinical study suggests that MK-2206's antiproliferative effect may be useful for NPC treatment; however, strategies for reinforcing this effect are needed to maximize clinical benefit.

Keywords: AKT inhibitor, MK-2206, nasopharyngeal carcinoma

\section{Introduction}

Nasopharyngeal carcinoma (NPC), a squamous cell carcinoma arising from the epithelium lining of the posterior nasopharynx, although rare in most parts of the world, is particularly common in Southern China and Southeast Asia ${ }^{1}$ and has caused very serious health problems in these areas. NPC is highly sensitive to radiation and chemotherapy. ${ }^{2}$ However, even with combined radiation and chemotherapy treatment, the prognosis for the metastatic form of NPC is not ideal, with disease relapse rates as high as $82 \% .^{3,4}$ Therefore, there is an urgent need to improve NPC treatment, especially targeted therapy.

The epidermal growth factor receptor (EGFR) represented a promising target against advanced NPC. Gefitinib, an oral quinazoline, is a highly selective EGFR-tyrosine kinase inhibitor. ${ }^{5}$ However, phase 2 studies of patients with metastatic or locoregional recurrent nasopharyngeal carcinoma found limited activity of gefitinib in recurrent NPC. ${ }^{6,7}$ A preclinical 
study suggested that persistent Protein kinase B (AKT) activation in NPC may be an important reason for resistance to gefitinib. ${ }^{8}$ AKT (a serine/threonine kinase v-AKT murine thymoma viral oncogene homolog), also called protein kinase, which is an important downstream target of the phosphatidylinositol-3 $\mathrm{OH}$ kinase (PI3K), helps regulate cell proliferation, differentiation, apoptosis, glucose metabolism, and tumorigenesis. ${ }^{9,10}$ In $\mathrm{NPC}$, the PI3K/AKT signaling pathway plays an important role in pathogenesis, and AKT promotes cell proliferation and survival. ${ }^{11,12}$ AKT can be deregulated through three different mechanisms: ${ }^{13}$ latent membrane proteins 1 can directly abnormally activate PI3K, leading to AKT phosphorylation, ${ }^{14}$ and AKT can also be directly abnormally activated by latent membrane proteins $2 \mathrm{~A}^{15}$ and decreased levels of phosphatase and tensin homolog, which are partially responsible for the abnormal upregulation of the PI3K/AKT pathway in NPC. ${ }^{16}$ AKT is deregulated in NPC as described earlier; therefore, it could be a potential target for cancer treatment.

MK-2206 is an orally active allosteric AKT inhibitor with half maximal inhibitory concentration $\left(\mathrm{IC}_{50}\right)$ values in the nanomolar range and broad preclinical antitumor activity. It is equally potent toward purified recombinant human AKT1 $\left(\mathrm{IC}_{50}, 5 \mathrm{nmol} / \mathrm{L}\right)$ and AKT2 enzyme ( $\left.\mathrm{IC}_{50}, 12 \mathrm{nmol} / \mathrm{L}\right)$ and is approximately fivefold less potent against human AKT3 $\left(\mathrm{IC}_{50}, 65 \mathrm{nmol} / \mathrm{L}\right) .{ }^{17}$ Recently, it has entered clinical development. ${ }^{18}$ In this study, we evaluated the antitumor growth effect of MK-2206 as a single agent in vitro and in vivo to investigate whether $\mathrm{AKT}$ was a promising new therapeutic target for NPC.

\section{Materials and methods}

\section{Cell culture}

One well-differentiated human NPC cell line, CNE-1, and three poorly differentiated human NPC cell lines, CNE-2, HONE-1, and SUNE-1, which were supplied as a present by Professor MS Zeng from State Key Laboratory of Oncology in South China, People's Republic of China, were cultivated in Roswell Park Memorial Institute 1640 medium supplemented with $10 \%$ fetal bovine serum, penicillin (100 units/mL), and streptomycin (100 units $/ \mathrm{mL}$ ) in a $5 \% \mathrm{CO}_{2}$ humidified atmosphere at $37^{\circ} \mathrm{C}$. Logarithmically growing cells were used in the experiments.

\section{Reagents and drug preparation}

MK-2206 was obtained from Merck \& Co., Inc., (Whitehouse Station, NJ, USA). The chemical name of MK-2206 is 8[4-(1-aminocyclobutyl) phenyl]-9-phenyl-1, 2, 4-triazolo $[3,4-f]^{1}$ naphthyridin-3(2H)-one hydrochloride [1:1]. The stock solutions of MK-2206 were formulated in dimethyl sulfoxide, stored at $-20^{\circ} \mathrm{C}$, and diluted in fresh culture medium immediately before use for in vitro experiments. In vivo, 30\% Captisol (CyDex Pharmaceuticals, Inc., Lenexa, KS, USA) was used to dose the MK-2206.

\section{Cell proliferation assay}

The cells were seeded into 96-well plates at an appropriate density per well. Twenty-four hours after plating, varying concentrations of MK-2206 were added to the wells. Cell proliferation was determined by using the Cell Counting Kit 8 (Dojindo, Japan) at 72 or 96 hours after dosing. The optical density was measured at $450 \mathrm{~nm}$ on an enzyme-linked immunosorbent assay reader (SpectraMax M5; Molecular Devices, Sunnyvale, CA, USA). The $\mathrm{IC}_{50}$ value was determined as the concentration resulting in 50\% cell growth inhibition after a 72 or 96 hours exposure to the drug compared with untreated control cells. All experiments were performed in triplicate in at least three independent experiments.

\section{Colony formation assay}

Single-cell suspension at a density of 500 cells per well was seeded in six-well plates. On the second day, cells were treated with the indicated concentrations of MK-2206. The medium was replaced with fresh medium containing MK-2206 every 3 days. After 10 days, the medium was removed and the plates were stained for the formation of cell colonies with methylene blue trihydrate. The picture of the colonies was then taken using a digital camera.

\section{Cell cycle analysis}

Cells were grown in six-well plates, and after reaching 70\% confluence, they were exposed to various concentrations of MK-2206 for 12, 24, and 48 hours. Cells were harvested by trypsinization, fixed with $70 \%$ ethanol, and stored at $-20^{\circ} \mathrm{C}$ overnight. Cell nuclei were stained for 30 minutes in the dark with a solution containing RNase $(0.2 \mathrm{mg} / \mathrm{mL})$ and propidium iodide $(0.05 \mathrm{mg} / \mathrm{mL})$. Analysis was performed using a FACScan flow cytometer (Cytomics ${ }^{\mathrm{TM}}$ FC 500; Beckman Coulter Inc., Brea, CA, USA), and a total of 20,000 stained nuclei were analyzed. Cell cycle data were processed with CELL Quest software (Becton, Dickinson, and Company, Franklin Lakes, NJ, USA). All experiments were performed in triplicate in at least three independent experiments.

\section{Detection of cell apoptosis}

The apoptosis in tumor cells was evaluated by Annexin V-fluorescein isothiocyanate (FITC) apoptosis assay and by diamidino-2-phenylindole (DAPI) nuclear staining assay. For the Annexin V-FITC apoptosis assay, the cells were collected 
and washed with phosphate-buffered saline after exposure to MK-2206 at indicated concentrations for indicated times. Annexin V-FITC and propidium iodide were used to determine the phosphatidylserine exposure on the outer plasma membrane. Flow cytometry analysis was performed using a Becton Dickinson FACScan.

For the DAPI nuclear staining assay, the cells on the coverglass in six-well plates were fixed with buffered formalin for 10 minutes, washed in ice cold phosphate-buffered saline, and soaked with DAPI staining solution in the dark. Then, the cover slips were put onto slides and the morphologic changes of apoptosis-characteristic nuclei were examined by fluorescent microscopy. Each test was repeated in triplicate.

\section{Western blot analysis}

After being treated with MK-2206, cells were harvested and lysed in cell lysis buffer (Cell Signaling Technology, Beverly, MA, USA). Proteins were resolved on sodium dodecyl sulfate polyacrylamide gel electrophoresis and transferred onto polyvinylidene fluoride membrane (F Hoffman-La Roche, Basel, Switzerland). The membranes were incubated overnight at $4^{\circ} \mathrm{C}$ with the following antibodies from Cell Signaling Technologies: total AKT, phospho-AKT (S473), phospho-AKT (T308), phospho-glycogen synthase kinase (GSK)-3 $\alpha / \beta$ (S21/9), phospho-proline-rich-Akt substrate, 40kDA (PRAS40) (T246), phospho-S6 Ribosomal Protein (S235/236), and phosphoTuberin/tuberous sclerosis complex2 (T1462), light chain 3 (LC3), glyceraldehyde 3-phosphate dehydrogenase. After being incubated with an appropriate horseradish peroxidaseconjugated secondary antibody for 1 hour at room temperature, bands were detected using an enhanced chemiluminescence system (Cell Signaling Technology).

\section{Electron microscopy}

CNE-2 cells were cultured in the presence of media or MK-2206 (5 and $2.5 \mu \mathrm{mol} / \mathrm{L}$, respectively) for 24 hours at $37^{\circ} \mathrm{C}$. Cells were harvested with trypsin ethylenediaminetetraacetic acid and fixed with $2.0 \%$ paraformaldehyde and $2.5 \%$ glutaraldehyde in 0.1 M sodium cacodylate buffer ( $\mathrm{pH} 7.4$ ). After fixation, samples were treated with $2 \%$ osmium tetroxide in $0.1 \mathrm{M}$ sodium cacodylate buffer, dehydrated through a graded ethanol series, and embedded in resin. Ultrathin sections were placed on formvar-coated slot copper grids and then counterstained in uranyl acetate and Reynolds lead citrate. Sections were finally viewed with an electron microscope (Tecnai G Spirit TWIN; FEI, Hillsboro, OR, USA).

\section{In vivo antitumor activity}

All animal experiments were conducted according to the National Institutes of Health Guide for the Care and Use of
Laboratory Animals and the UK Coordinating Committee on Cancer Research. Four- to 6-week-old male BALB/c nude mice were purchased from Hunan Slac Jingda Laboratory Animal Co., Ltd., (Hunan, People's Republic of China). CNE-2 cells at $1 \times 10^{7} / \mathrm{mL}$ were suspended in serum-free medium, and $0.2 \mathrm{~mL}$ medium was injected subcutaneously into the right flank of each nude mouse. When mean tumor volume reached approximately $50 \mathrm{~mm}^{3}$, the mice were randomized into three groups ( $\mathrm{n}=7$ /group) with approximately equivalent ranges of tumor volume between groups. The MK-2206 was dosed with 30\% Captisol (Cydex). MK-2206 (240 mg/kg, three times a week), MK-2206 (480 mg/kg, once a week), and 30\% Captisol (Cydex) diluents were administered by oral gavage for 2 weeks for each group. Tumor growth was measured with calipers every other day, and the tumor volume was calculated by the following formula: volume $\left(\mathrm{mm}^{3}\right)=$ length $\times$ width $^{2} \times 0.5$.

Mice were killed when mean tumor volume in the control group was larger than $2,000 \mathrm{~mm}^{3}$. Harvested tumor specimens were weighed and fixed in $10 \%$ buffered formalin and embedded in paraffin.

\section{Immunohistochemical staining}

Immunohistochemical staining was done on formalinfixed, paraffin-embedded tissue sections of tumor excised from xenografts by using the standard avidinbiotin complex-peroxidase method, as described in our previous paper. ${ }^{19}$ Tumor sections were stained with anti-p-PRAS40 (T246), phospho-S6 ribosomal protein (S235/236).

\section{Statistical analysis}

Cell culture-based assays were repeated at least three times; data are expressed as mean values \pm standard deviation. One-way analysis of variance, followed by Dennett's test, was used to compare the percentages of cells in G1 phase under various concentrations of MK-2206, with the control group. Comparisons for mean final tumor volumes in the xenograft studies were analyzed by one-way analysis of variance, and $P$-values of pairwise comparisons were adjusted with Bonferroni correction. Two-sided $P<0.05$ was considered statistically significant. Statistical analysis was performed using SPSS version 16.0 software (IBM, Armonk, NY, USA).

\section{Results}

Proliferation inhibitory effects of MK-2206 The NPC cell lines CNE-1, CNE-2, HONE-1, and SUNE-1 were treated with increasing doses of MK-2206 (0-10 $\mu \mathrm{mol} / \mathrm{L})$ 
for 72 and 96 hours. As shown in Figure 1A-D, exposure to MK-2206 resulted in dose- and time-dependent inhibition of cell viability. At 72 and 96 hours, the $\mathrm{IC}_{50}$ values of MK-2206 in CNE-1, CNE-2, and HONE-1 cell lines were 3-5 $\mu \mathrm{M}$, and in SUNE-1, they were less than $1 \mu \mathrm{M}$ (Figure 1E). In the long-term colony formation assay, we obtained similar results; that is, that MK-2206 reduced the sizes of the colonies in a dose-dependent manner (Figure 1F).

\section{A}

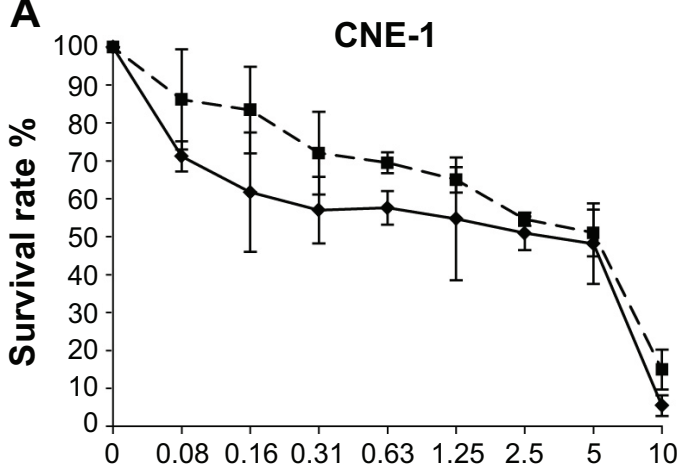

Concentration of MK-2206 ( $\mu \mathrm{M})$

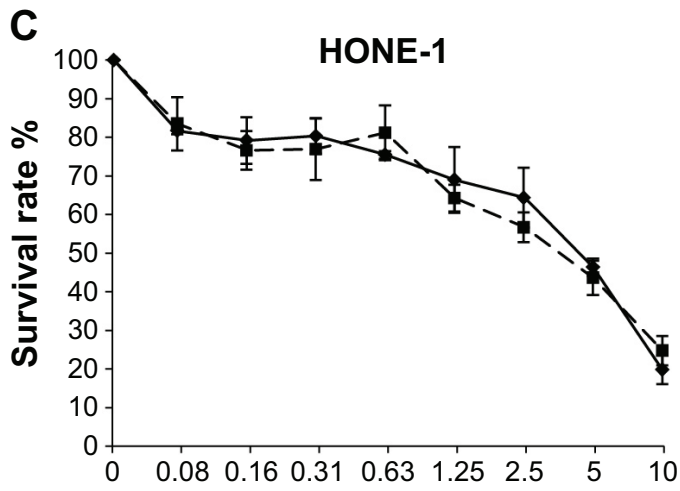

Concentration of MK-2206 ( $\mu \mathrm{M})$

\section{MK-2206 induced cell cycle arrest} at GI phase

The effects of MK-2206 on the cell cycle progression in poorly differentiated human NPC cell lines CNE-2, HONE-1, and SUNE-1 were determined by flow cytometry. In CNE-2 and HONE-1 cells, treatment with various concentrations of MK-2206 for 24 and 48 hours resulted in a dose-dependent increase in the percentage of cells in

B

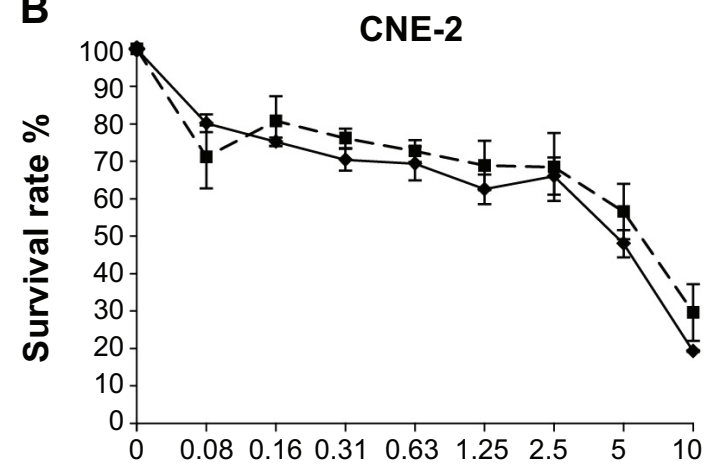

Concentration of MK-2206 ( $\mu \mathrm{M})$

D

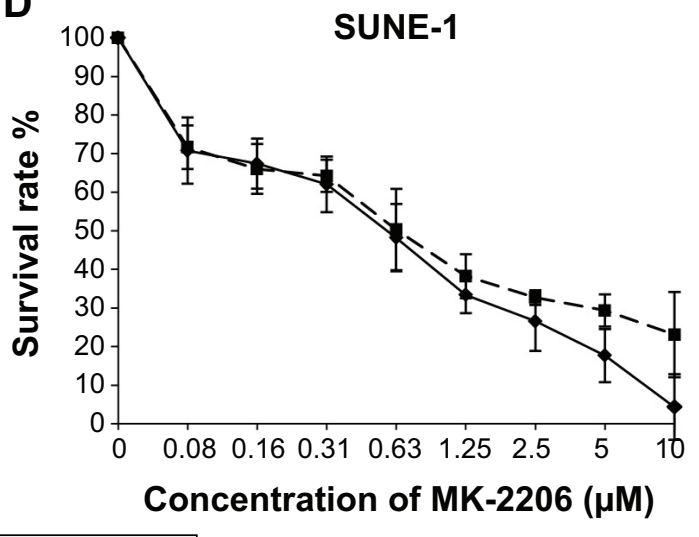

E
\begin{tabular}{|l|c|c|}
\hline & IC $_{50}$ of MK-2206 $(\boldsymbol{\mu M})$ \\
\cline { 2 - 3 } & $\mathbf{7 2}$ hours & $\mathbf{9 6}$ hours \\
\hline CNE-1 & $4.66 \pm 1.41$ & $2.96 \pm 1.47$ \\
CNE-2 & $5.99 \pm 2.26$ & $4.53 \pm 1.08$ \\
HONE-1 & $3.54 \pm 2.15$ & $3.37 \pm 0.84$ \\
SUNE-1 & $0.68 \pm 0.40$ & $0.52 \pm 0.18$ \\
\hline
\end{tabular}

Figure I Growth-inhibitory effect of MK-2206 on nasopharyngeal carcinoma cell lines.

Notes: (A) CNE-I. (B) CNE-2. (C) HONE-I. (D) SUNE-I. Cells were cultured at 2,500-4,000 cells per well in a 96-well plate, exposed to different concentrations of MK2206, and incubated for 72 and 96 hours. Points, average of three experiments; bars, standard error; solid line, 96 hours test; dashed line, 72 hours test. (E) Average half maximal inhibitory concentration for cell growth in nasopharyngeal carcinoma cell lines after exposure to MK-2206 for 72 and 96 hours. (F) Cells at a density of 500 cells per well were seeded in six-well plates. On the second day, cells were treated with the indicated concentrations of MK-2206. The same treatments were repeated every 3 days. After 10 days, the plates were stained for the formation of cell colonies with methylene blue trihydrate. The picture of the colonies was then taken using a digital camera. One representative experiment is shown (HONE-I). Values are presented as mean \pm standard deviation.

Abbreviations: $I_{50}$, half maximal (50\%) inhibitory concentration; CON, control. 
G0/G1 phase and a concomitant reduction of cell numbers in $\mathrm{S}$ phase. One-way analysis of variance followed by Dennett's test was used to compare the percentages of cells in G1 phase under various concentrations of MK-2206 with the control group. Statistical significance $(P<0.05)$ was observed when cells (CNE-2 and HONE-1) were treated with MK-2206 5 and $10 \mu \mathrm{mol} / \mathrm{L}$ for 24 hours and HONE-1 with MK-2206 $10 \mu \mathrm{mol} / \mathrm{L}$ for 48 hours (Figure 2).

G1 arrest was also observed in SUNE-1 cell lines after being treated with MK-2206, although the trend was not as obvious as that in CNE-2 and HONE-1. Using HONE-1 and CNE-2 cells for subsequent studies, we next investigated the temporal pattern of $\mathrm{G} 1$ arrest after treatment with MK-2206 for 12 and 72 hours and found the G1 arrest was most typical in 24 hours treatment of MK-2206.

\section{MK-2206 does not induce apoptosis}

In our study, we also examined whether MK-2206 was capable of inducing apoptosis in NPC cells. Cell morphology stained with DAPI was observed to identify the apoptotic cell population.
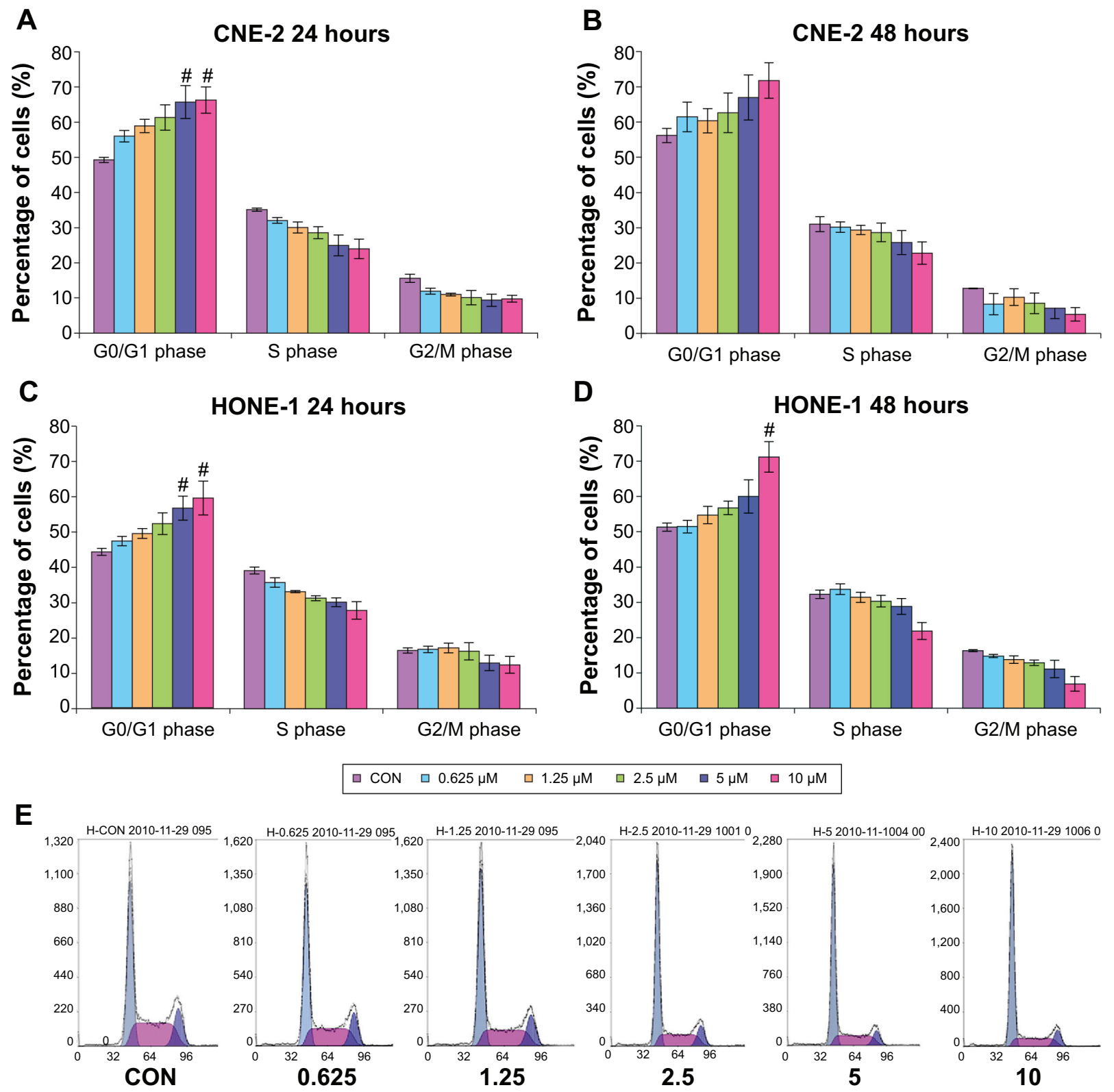

Figure 2 MK-2206 induces cell cycle arrest at GI in a dose-dependent manner in CNE-2 and HONE-I cells.

Notes: ${ }^{\#}<0.05$ compared with vehicle control. (A) CNE-2 (24 hours). (B) CNE-2 (48 hours). (C) HONE-I (24 hours). (D) HONE-I (48 hours). Cell lines were incubated with 0-10 $\mu$ M MK-2206 for 24 or 48 hours, respectively, and were thereby labeled with propidium iodine, followed by analysis with flow cytometry. Values are presented as mean \pm standard deviation. (E) One representative experiment is shown (HONE-I 24 hours). Abbreviation: CON, control. 
Treatment with MK-2206 for 24 and 48 hours did not appreciably induce apoptosis in NPC cell lines. One representative experiment is shown in Figure 3A (CNE-2 24 hours).

To further confirm our results, Annexin V-FITC/ propidium iodide double-staining assay was used to detect the apoptotic cells. Interestingly, no obvious increase in apoptotic cell death was detected when treated with an increasing dose of MK-2206 for 24, 48, or even 72 hours (Figure 3B).

\section{MK-2206 inhibited phosphorylation of AKT downstream targets}

GSK-3 is a substrate of PI3K/AKT. ${ }^{20}$ Proline-rich Akt substrate of $40 \mathrm{kDa}$ (PRAS40) is also one of the novel downstream target substrates of PI3K/AKT. ${ }^{21}$ Phosphorylated PRAS40 can mediate Akt signals to the mammalian target of rapamycin (mTOR) complex, followed by phosphorylation of the ribosomal protein S6. ${ }^{22}$ That is to say, PRAS40,

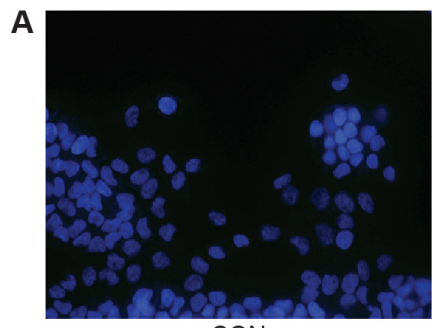

CON

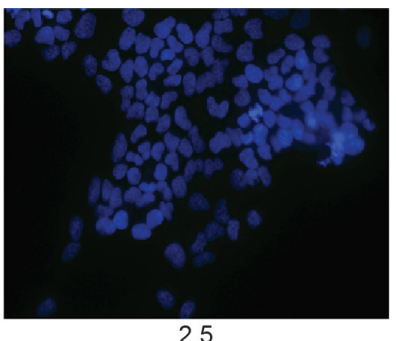

2.5

B

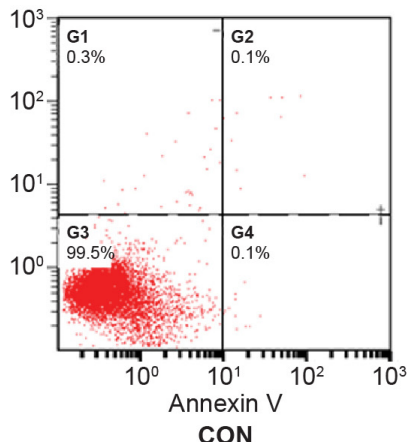

CON

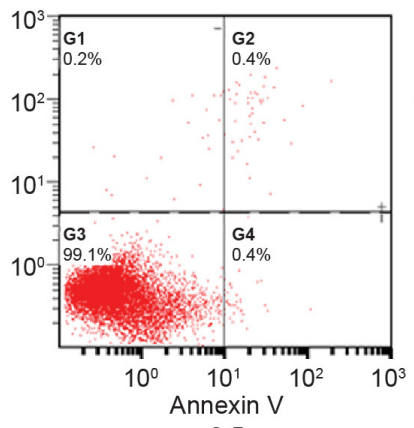

2.5

\section{CNE-2 24 hours}

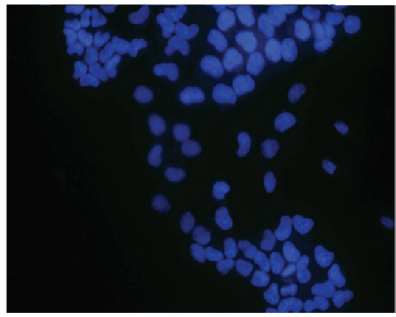

0.625
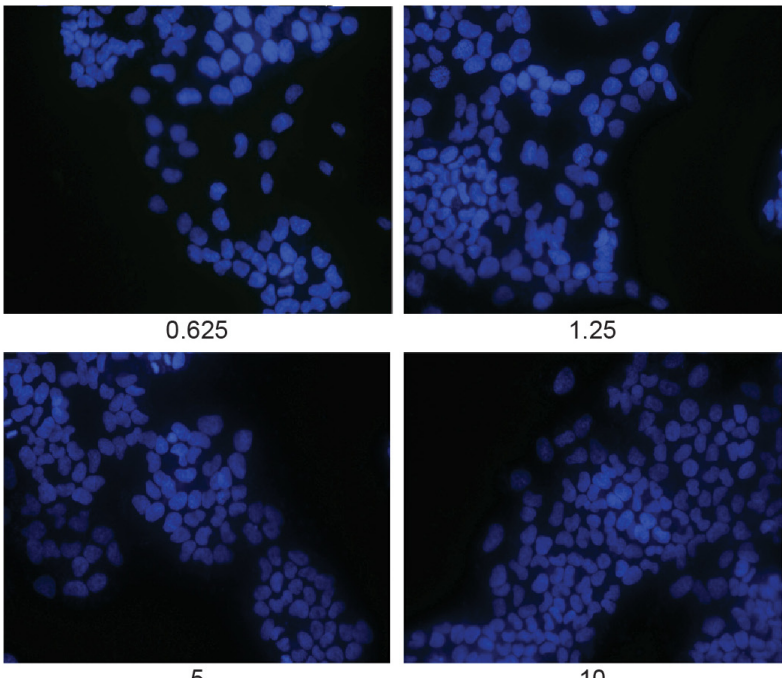

1.25

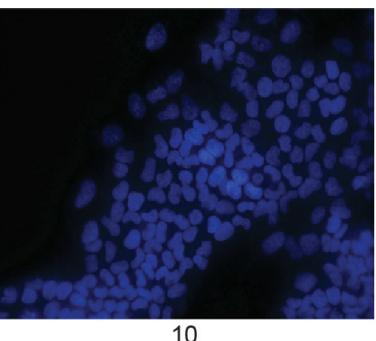

CNE-2 72 hours

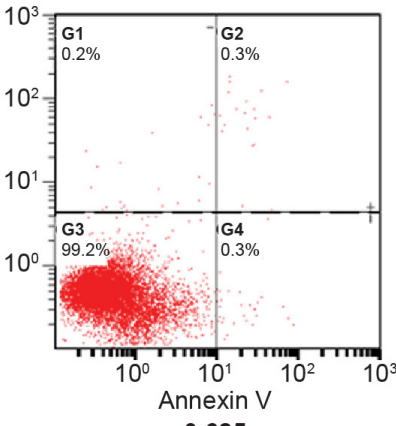

0.625

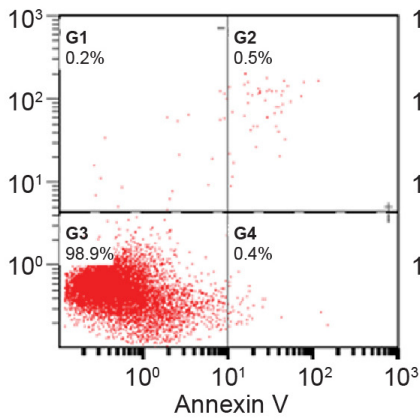

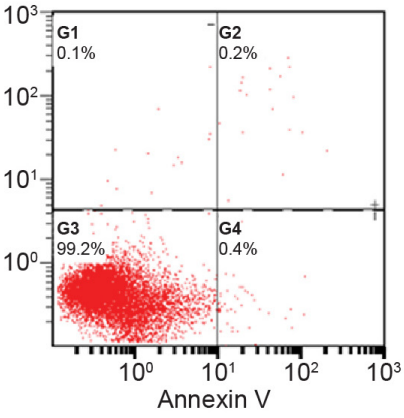

1.25

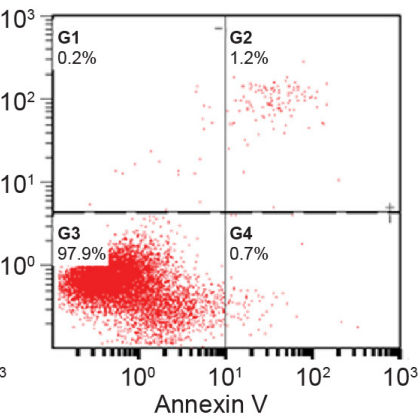

10

Figure 3 No apoptosis was induced by MK-2206 in the four nasopharyngeal carcinoma cell lines.

Notes: (A) No characteristic apoptotic cells were found in diamidino-2-phenylindole nuclear staining assay. Magnification, $\times 40$. The experiments are repeated twice with similar results. (B) No evidence of apoptosis in any of the four cell lines was seen. Testing was by Annexin $V$ assays, which were carried out 72 hours after a single treatment of cells with MK-2206 at doses as high as $10 \mu \mathrm{M}$. One representative experiment is shown (CNE-2 72 hours).

Abbreviation: $\mathrm{CON}$, control. 
GSK $\alpha / \beta$, and S6 are important downstream target substrates of the PI3K/AKT signaling pathway.

We examined whether phosphorylation of downstream targets of AKT could be inhibited by MK-2206 in SUNE-1 and CNE-2 cell lines. As shown in Figure 4, after exposure to MK-2206 for 24 hours, the phosphorylation levels of PRAS40 and S6 were attenuated by MK-2206 in a dose-dependent manner. However, we did not see changes of phosphorylation of GSK $\alpha / \beta$ and AKT in our study.

\section{MK-2206 induce autophagy in NPC cells}

Autophagy is regulated by the AKT/phosphatidylinositol 3-kinase and mTOR pathways, ${ }^{23}$ so we examined whether MK-2206 treatment triggers autophagy in NPC cells. Microtubule-associated protein $1 \mathrm{LC} 3$, an essential autophagy protein, is a human homolog of the yeast ATG8. ${ }^{24}$ It is processed from a full-length protein (MAP-LC3-I) to a cleaved and lipidated form (LC3-II) during autophagy, and LC3-II has been used as a specific marker for autophagy. ${ }^{24,25}$ As shown in Figure 5A, after treatment with MK-2206 for 24 hours, the appearance of LC3-II in CNE-2 cells increased dose-dependently.

Formation of double membrane vacuoles that sequester organelles is also characteristic of autophagy. ${ }^{24}$ Electron microscopy visualized abundant double or multimembrane vacuoles in the cytoplasm of MK-2206-treated cells. In contrast, these vacuoles were rarely seen in the control cells (Figure 5B).

\section{Antitumor activity of MK-2206 in vivo}

We evaluated the efficacy of MK-2206 in CNE-2 xenografts in nude mice. In this experiment, we used two different doses of MK-2206. As presented in Figure 6A, compared with the control group (30\% Captisol diluent), both MK-2206 doses $(480 \mathrm{mg} / \mathrm{kg}$ once a week and $240 \mathrm{mg} / \mathrm{kg}$ three times a week) can inhibit the growth of human CNE-2 xenografts in nude mice. At the end of the experiment, we obtained identical results by directly measuring tumor weights. In the two MK-2206 groups, the tumor weights were much lighter than the control group $(P<0.01$; Figure 6B). Temporal body weight reduction was observed after receiving the MK-2206 treatment (Figure 6C). No other obvious toxicity was observed in mice.

Given the therapeutic efficacy of MK-2206 in our in vivo model, we examined the p-PRAS40 and p-S6 expressions in tumor samples. As shown in Figure 6D, phosphorylation levels of PRAS40 and S6 in the tumor were also inhibited by MK-2206.

\section{Discussion}

In the current study, in vitro dose-response curves showed that inhibition of AKT signaling with the allosteric inhibitor MK-2206 could attenuate cell growth. Furthermore, the $\mathrm{IC}_{50}$ value of MK-2206 was less than $1 \mu \mathrm{mol} / \mathrm{L}$ in SUNE-1 cell lines and was $3-5 \mu \mathrm{mol} / \mathrm{L}$ in the other three NPC cell lines. These results confirmed findings from previous studies of MK-2206 on head and neck squamous cell carcinoma ${ }^{26}$ and
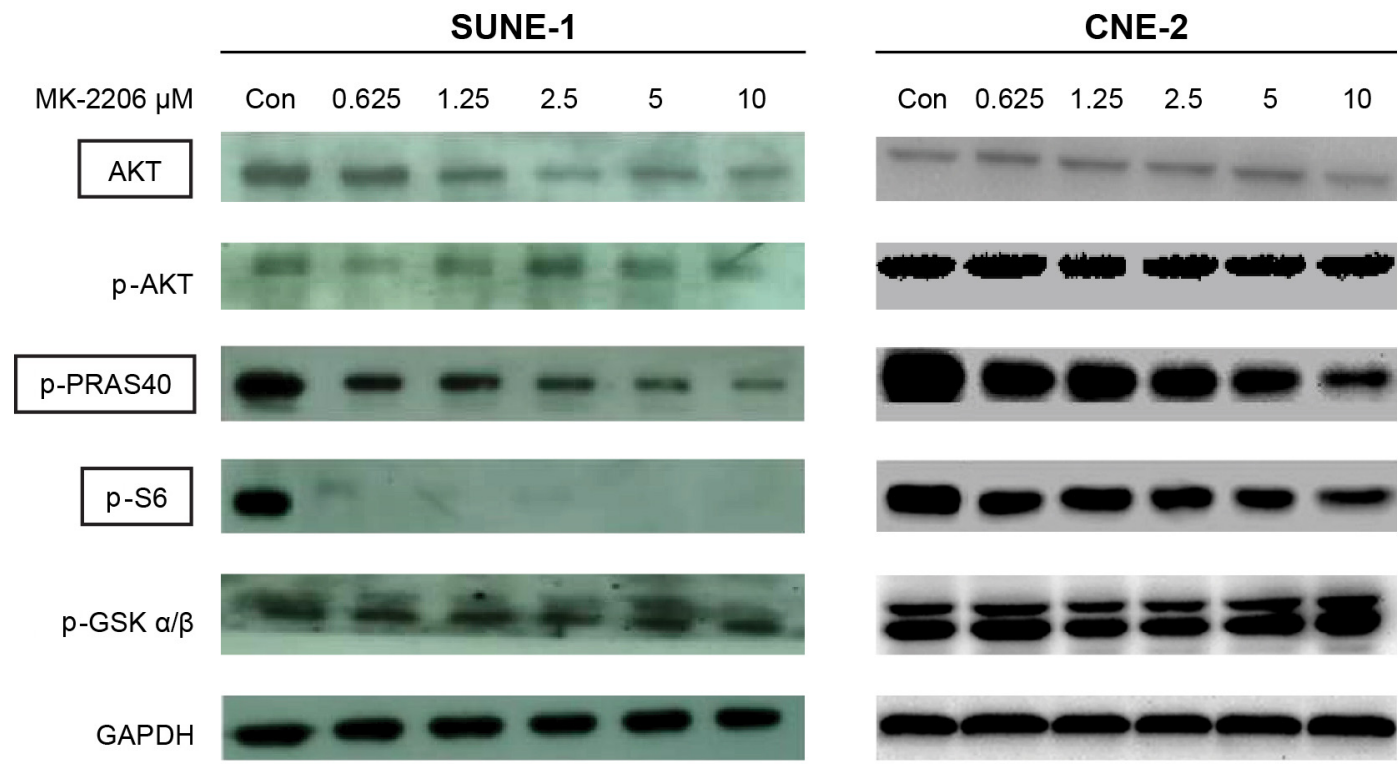

Figure 4 MK-2206 inhibited phosphorylation of AKT downstream targets.

Notes: SUNE-I and CNE-2 cells were treated with different concentrations of MK-2206 for 24 hours. Then the cells were collected and lysed. Western blot was conducted and probed with anti-AKT, anti-phospho-AKT, anti-phospho-PRAS40, anti-phospho-S6, and anti-phospho-GSK $\alpha / \beta$.

Abbreviations: AKT, Protein kinase B; Con, control; GSK, glycogen synthase kinase; PRAS40, proline-rich-Akt substrate, 40kDA. 
A

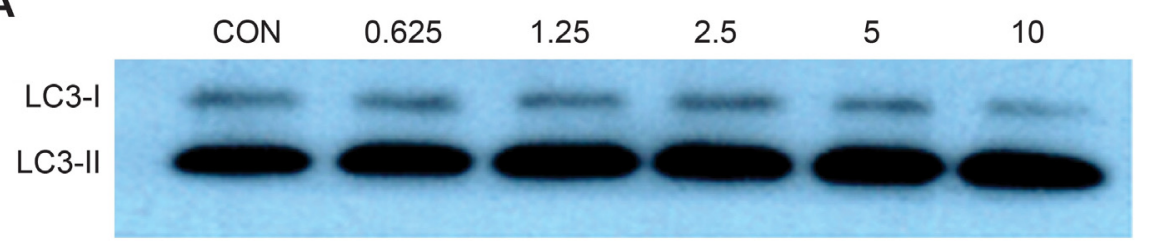

GAPDH

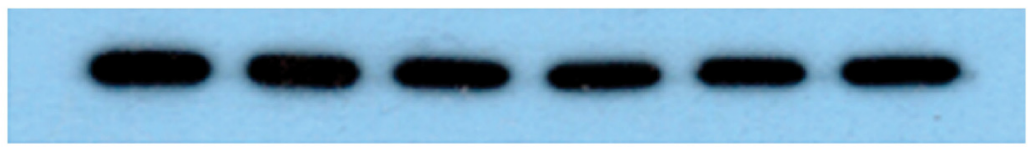

B

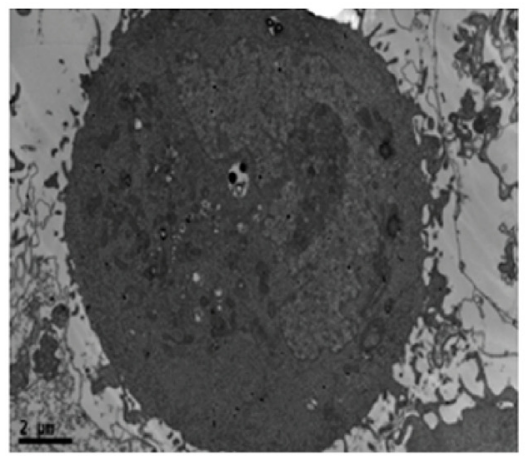

CON 5,800 X

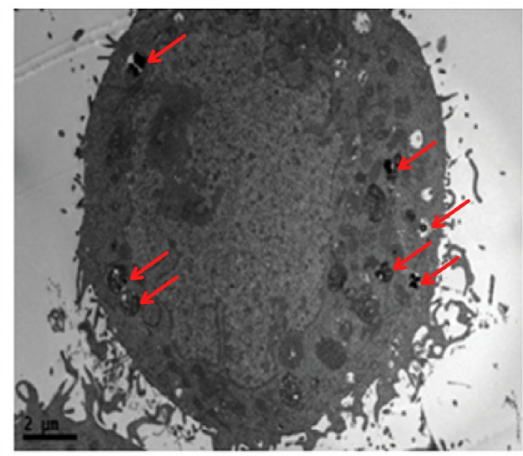

MK-2206 $2.5 \mu \mathrm{mol} / \mathrm{L}$ $5,800 \times$

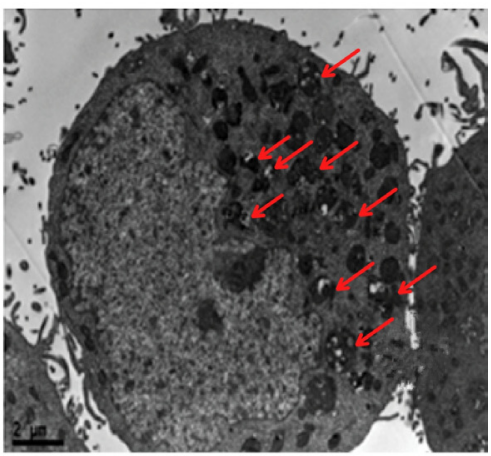

MK-2206 $5 \mu \mathrm{mol} / \mathrm{L}$ $5,800 \times$

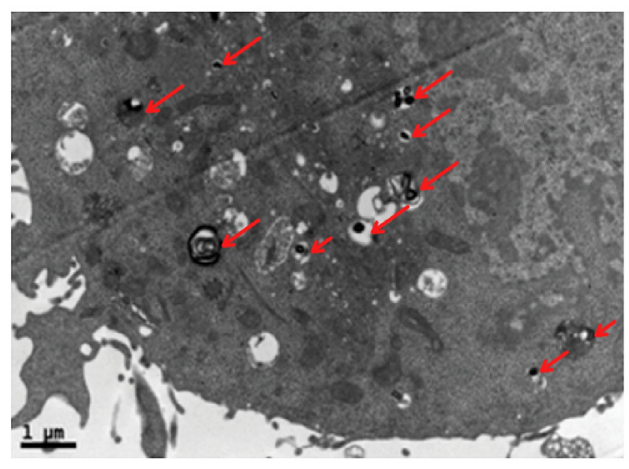

MK-2206 $2.5 \mu \mathrm{mol} / \mathrm{L}$ $7,900 \mathrm{X}$

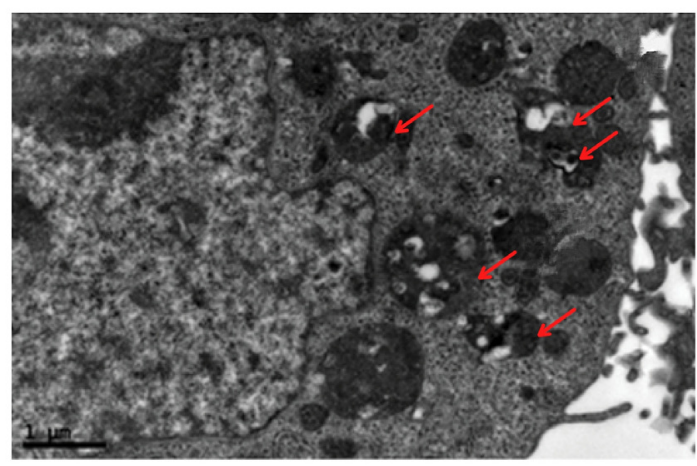

MK-2206 $5 \mu \mathrm{mol} / \mathrm{L}$

$13,500 \mathrm{X}$

Figure 5 Effect of MK-2206 on autophagy in human nasopharyngeal carcinoma cells.

Notes: (A) CNE-2 cells cultured in medium supplemented with 10\% fetal bovine serum were treated with MK-2206 for 24 hours; the level of light chain 3 was examined by Western blot. Glyceraldehyde 3-phosphate dehydrogenase was used as a loading control. (B) CNE-2 cells treated with MK-2206 (5 $\mu$ mol/L, $2.5 \mu \mathrm{mol} / \mathrm{L})$ or vehicle were harvested by trypsinization, fixed, and embedded in spur resin. Ultrathin sections were cut and examined by transmission electron microscope. Arrows indicate autophagic vacuoles.

Abbreviation: CON, control.

NPC. ${ }^{27}$ However, our in vivo data confirmed for the first time that AKT inhibition by MK-2206 could inhibit the growth of CNE-2 NPC cells subcutaneously implanted into nude mice.

Our in vitro data showed that MK-2206 reduced the phosphorylation levels of PRAS40 and S6 but did not change that of GSK $\alpha / \beta$. This downstream regulation was also demonstrated in our in vivo model. PRAS40 is one of the novel downstream target substrates of the PI3K/AKT signaling pathway. ${ }^{21}$ Phosphorylation of PRAS40 at Thr246 by AKT facilitates efficient phosphorylation of Ser183 by mTORC1, which further results in phosphorylation of 4EBP1 and S6K1, followed by phosphorylation of the ribosomal protein $\mathrm{S} 6 .{ }^{22}$ On the basis of these findings, we hypothesize that inhibition through the AKT/mTOR pathways seems to be the mechanism for NPC cell growth inhibition. In a clinical trial of MK-2206, immunofluorescence studies in hair follicles also indicated that the pThr246 signal on PRAS40 was effectively blocked. ${ }^{18}$

This trial, in patients with advanced solid tumors, also established that the maximum tolerated dose of MK-2206 administered on alternate days was $60 \mathrm{mg} .{ }^{18}$ 
A

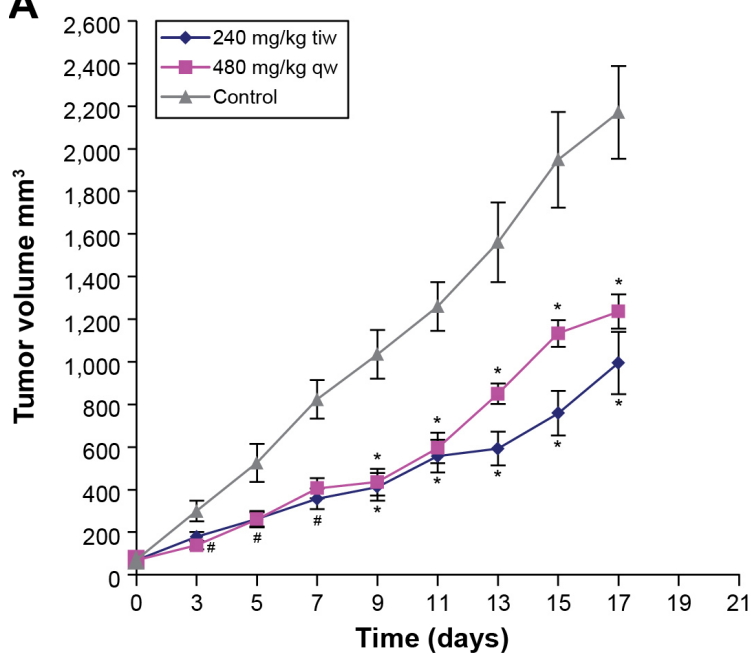

C

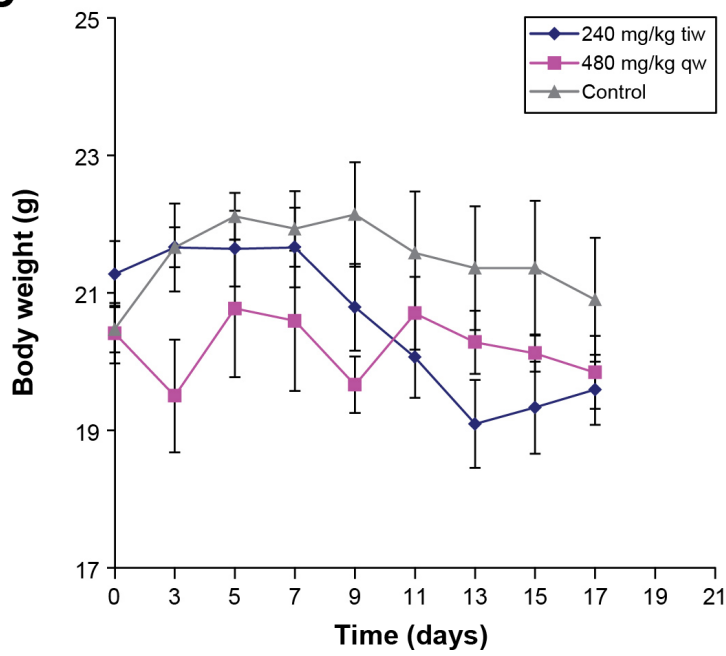

B

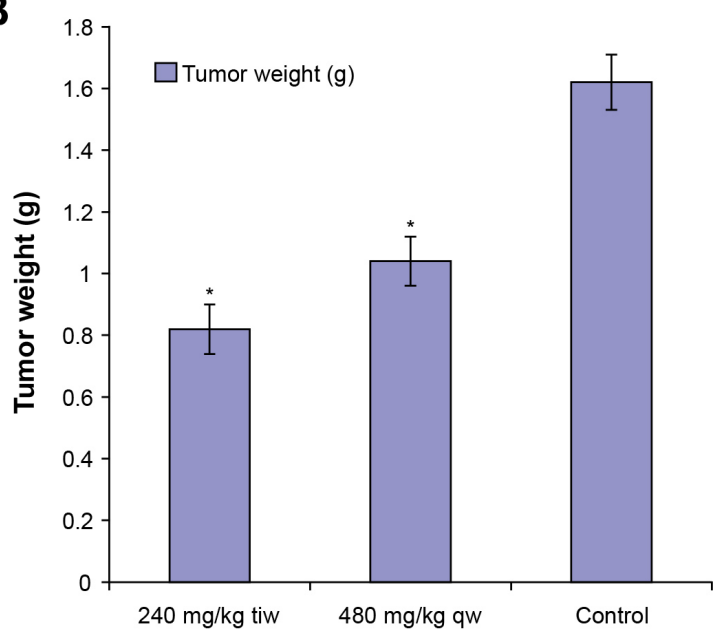

D
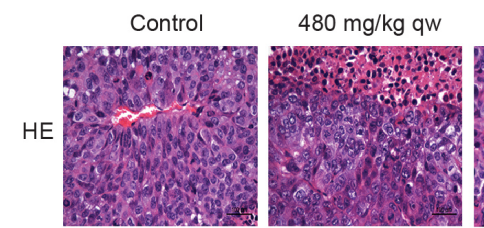

$240 \mathrm{mg} / \mathrm{kg}$ tiw

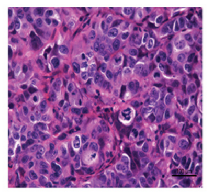

p-PRAS40
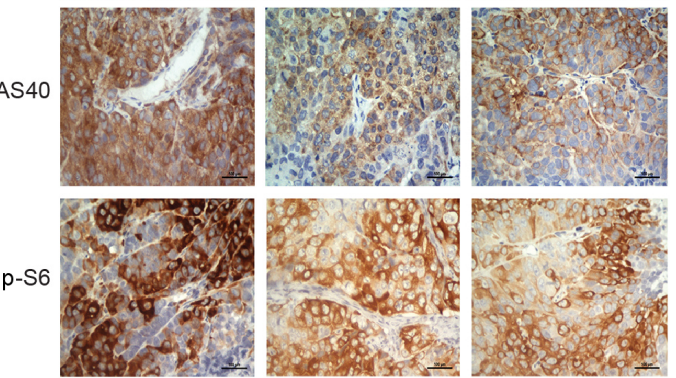

Figure 6 Effects of MK-2206 on tumor growth of human CNE-2 xenografts in nude mice.

Notes: (A) The treatments began on day I after grouping (day 0), including 30\% Captisol $10 \mathrm{~mL} / \mathrm{kg}$ once a week, MK-2206 $480 \mathrm{mg} / \mathrm{kg}$ once a week, and MK-2206 $240 \mathrm{mg} / \mathrm{kg}$ three times a week for 2 weeks. During treatment, tumor volumes were measured every other day. Points, mean of tumors; bars, standard deviation. $\# P<0.05$ compared with vehicle control; $* P<0.01$ compared with vehicle control. (B) After I 8 days of grouping, the mice were killed and the tumors were removed and weighed. $* P<0.01$ compared with vehicle control. (C) Body weight was measured every other day and used to assess toxicity of treatment. (D) Tumors were resected, fixed, and paraffin embedded. The sections were analyzed by hematoxylin-eosin ( $\mathrm{HE})$ staining and by immunohistochemistry, using p-PRAS40 and p-S6 antibodies. The representative photographs in tumor sections are shown $(\times 400)$.

Abbreviations: tiw, three times per week; qw, once a week; PRAS40, proline-rich-Akt substrate, 40kDA.

Mean plasma concentration-time profiles after the first and last cycle 1 doses ( $60 \mathrm{mg}$, every other day) are less than $100 \mathrm{nM}$, which was slightly lower than the $\mathrm{IC}_{50}$ achieved in our in vitro study. Although preclinical data showed that MK-2206 monotherapy can inhibit the growth of NPC, clinical data suggest it may not be as effective as we expected. Strategies for reinforcing the activity of the AKT inhibitors are needed to make this type of drug more valuable.

We observed that a single treatment of MK-2206 can cause cell cycle arrest at the G1 phase in the NPC cell lines we tested. Cyclin D1, a cyclin required for G1 to S transition, which was found to be reduced on MK-2206 treatment, could be the main cause for the G1 block. ${ }^{28}$ However, it rarely induces any apoptosis. Because AKT/phosphatidylinositol 3-kinase and mTOR pathways are related to autophagic activity, ${ }^{23} \mathrm{we}$ supposed that this may be another possible mechanism of NPC cancer cell growth inhibition by MK-2206. As shown in our results, MK-2206 indeed caused autophagy in NPC cell lines, which was consistent with previous reports that autophagy is activated when AKT is inhibited. ${ }^{29}$

Although activation of autophagy (also termed type 2 programmed cell death) can promote autophagic cell death, this form of intracellular degradative process also has been noted to support cell survival. ${ }^{30} \mathrm{Cheng}$ and colleagues reported that promoting the switch from autophagy to apoptotic cell death can enhance the effectiveness of MK-2206 against 
glioma. ${ }^{31}$ In NPC, the functional associations of autophagy with apoptosis in response to AKT inhibition remain largely unknown. Thus, whether suppressing autophagy would reinforce the antitumor activity needs to be clarified. Further evaluation is currently under way.

Only using four cell lines is one of the limitations of our study. It is not known whether other cell lines would respond differently to MK-2206 or yield additional insights in the differential response to AKT inhibition. Furthermore, there are potentially multiple strategies for reinforcing the activity of the AKT inhibitors that have yet to be tested. Despite these limitations, this study is one of very few preclinical studies that describe the use of AKT inhibition in NPC, suggesting a potential role for MK-2206 in the treatment of NPC in the future.

\section{Acknowledgments}

Martha Carroll Vollmer, MS, from Merck \& Co., Inc., Whitehouse Station, NJ, USA, provided editorial assistance in the preparation of this manuscript. This work was also supported by National High Technology Research and Development Program of China (2012AA02A501).

\section{Disclosures}

Michael Lam and Li Yan are employed by Merck \& Co., Inc. Merck supplied the drug MK-2206 used in this study but did not participate in the development of the protocol, the running of the trial, or analysis of results. The authors report no other conflicts of interest in this work.

\section{References}

1. Altomare DA, Testa JR. Perturbations of the AKT signaling pathway in human cancer. Oncogene. 2005;24(50):7455-7464.

2. Altun M, Fandi A, Dupuis O, Cvitkovic E, Krajina Z, Eschwege F. Undifferentiated nasopharyngeal cancer (UCNT): current diagnostic and therapeutic aspects. Int J Radiat Oncol Biol Phys. 1995;32(3): 859-877.

3. Cheng SH, Jian JJ, Tsai SY, et al. Long-term survival of nasopharyngeal carcinoma following concomitant radiotherapy and chemotherapy. Int $J$ Radiat Oncol Biol Phys. 2000;48(5):1323-1330.

4. Yamashita S, Kondo M, Hashimoto S. Squamous cell carcinoma of the nasopharynx. An analysis of failure patterns after radiation therapy. Acta Radiol Oncol. 1985;24(4):315-320.

5. Ranson M, Hammond LA, Ferry D, et al. ZD1839, a selective oral epidermal growth factor receptor-tyrosine kinase inhibitor, is well tolerated and active in patients with solid, malignant tumors: results of a phase I trial. J Clin Oncol. 2002;20(9):2240-2250.

6. Chua DT, Wei WI, Wong MP, Sham JS, Nicholls J, Au GK. Phase II study of gefitinib for the treatment of recurrent and metastatic nasopharyngeal carcinoma. Head Neck. 2008;30(7):863-867.

7. Ma B, Hui EP, King A, et al. A phase II study of patients with metastatic or locoregionally recurrent nasopharyngeal carcinoma and evaluation of plasma Epstein-Barr virus DNA as a biomarker of efficacy. Cancer Chemother Pharmacol. 2008;62(1):59-64.
8. Ma BB, Lui VW, Poon FF, et al. Preclinical activity of gefitinib in non-keratinizing nasopharyngeal carcinoma cell lines and biomarkers of response. Invest New Drugs. 2010;28(3):326-333.

9. Bellacosa A, Testa JR, Staal SP, Tsichlis PN. A retroviral oncogene, akt, encoding a serine-threonine kinase containing an SH2-like region. Science. 1991;254(5029):274-277.

10. Manning BD, Cantley LC. AKT/PKB signaling: navigating downstream. Cell. 2007;129(7):1261-1274.

11. Yip WK, Leong VC, Abdullah MA, Yusoff S, Seow HF. Overexpression of phospho-Akt correlates with phosphorylation of EGF receptor, FKHR and BAD in nasopharyngeal carcinoma. Oncol Rep. 2008;19(2): 319-328.

12. Mei YP, Zhou JM, Wang Y, et al. Silencing of LMP1 induces cell cycle arrest and enhances chemosensitivity through inhibition of AKT signaling pathway in EBV-positive nasopharyngeal carcinoma cells. Cell Cycle. 2007;6(11):1379-1385.

13. Chou J, Lin YC, Kim J, et al. Nasopharyngeal carcinoma - review of the molecular mechanisms of tumorigenesis. Head Neck. 2008;30(7): 946-963.

14. Mainou BA, Everly DN Jr, Raab-Traub N. Epstein-Barr virus latent membrane protein 1 CTAR 1 mediates rodent and human fibroblast transformation through activation of PI3K. Oncogene. 2005;24(46):6917-6924.

15. Morrison JA, Raab-Traub N. Roles of the ITAM and PY motifs of Epstein-Barr virus latent membrane protein $2 \mathrm{~A}$ in the inhibition of epithelial cell differentiation and activation of $\{$ beta $\}$-catenin signaling. J Virol. 2005;79(4):2375-2382.

16. Pedrero JM, Carracedo DG, Pinto CM, et al. Frequent genetic and biochemical alterations of the PI 3-K/AKT/PTEN pathway in head and neck squamous cell carcinoma. Int J Cancer. 2005;114(2): 242-248.

17. Yan L. MK-2206: a potent oral allosteric AKT inhibitor. Presented at: 100th Annual Meeting of the American Association for Cancer Research; April 18-22; 2009; Denver, CO. Abstract DDT01-1.

18. Yap TA, Yan L, Patnaik A, et al. First-in-man clinical trial of the oral pan-AKT inhibitor MK-2206 in patients with advanced solid tumors. J Clin Oncol. 2011;29(35):4688-4695.

19. Zhao YY, Xue C, Jiang W, et al. Predictive value of intratumoral microvascular density in patients with advanced non-small cell lung cancer receiving chemotherapy plus bevacizumab. J Thorac Oncol. 2012;7(1):71-75.

20. Wang H, Zhang Q, Wen Q, et al. Proline-rich Akt substrate of $40 \mathrm{kDa}$ (PRAS40): a novel downstream target of PI3k/Akt signaling pathway. Cell Signal. 2012;24(1):17-24.

21. Yap TA, Walton MI, Hunter LJ, et al. Preclinical pharmacology, antitumor activity, and development of pharmacodynamic markers for the novel, potent AKT inhibitor CCT128930. Mol Cancer Ther. 2011;10(2):360-371.

22. Meric-Bernstam F, Gonzalez-Angulo AM. Targeting the mTOR signaling network for cancer therapy. J Clin Oncol. 2009;27(13):2278-2287.

23. Takeuchi H, Kondo Y, Fujiwara K, et al. Synergistic augmentation of rapamycin-induced autophagy in malignant glioma cells by phosphatidylinositol 3-kinase/protein kinase B inhibitors. Cancer Res. 2005;65(8):3336-3346.

24. Wu H, Yang JM, Jin S, Zhang H, Hait WN. Elongation factor-2 kinase regulates autophagy in human glioblastoma cells. Cancer Res. 2006; 66(6):3015-3023.

25. Mizushima N. Methods for monitoring autophagy. Int J Biochem Cell Biol. 2004;36(12):2491-2502.

26. Knowles JA, Golden B, Yan L, Carroll WR, Helman EE, Rosenthal EL. Disruption of the AKT pathway inhibits metastasis in an orthotopic model of head and neck squamous cell carcinoma. Laryngoscope. 2011;121(11):2359-2365.

27. Ma BB, Lui VW, Hui CW, et al. Preclinical evaluation of the AKT inhibitor MK-2206 in nasopharyngeal carcinoma cell lines. Invest New Drugs. 2013;31(3):567-575. 
28. Jiao P, Zhou YS, Yang JX, et al. MK-2206 induces cell cycle arrest and apoptosis in HepG2 cells and sensitizes TRAIL-mediated cell death. Mol Cell Biochem. 2013;382(1-2):217-224.

29. Degtyarev M, De Mazière A, Orr C, et al. Akt inhibition promotes autophagy and sensitizes PTEN-null tumors to lysosomotropic agents. J Cell Biol. 2008;183(1):101-116.
30. Kundu M, Thompson CB. Macroautophagy versus mitochondrial autophagy: a question of fate? Cell Death Differ. 2005;12(suppl 2):1484-1489.

31. Cheng Y, Ren X, Zhang Y, et al. eEF-2 kinase dictates cross-talk between autophagy and apoptosis induced by Akt Inhibition, thereby modulating cytotoxicity of novel Akt inhibitor MK-2206. Cancer Res. 2011;71(7):2654-2663.

\section{Publish your work in this journal}

Drug Design, Development and Therapy is an international, peerreviewed open-access journal that spans the spectrum of drug design and development through to clinical applications. Clinical outcomes, patient safety, and programs for the development and effective, safe, and sustained use of medicines are a feature of the journal, which has also been accepted for indexing on PubMed Central. The manuscript management system is completely online and includes a very quick and fair peer-review system, which is all easy to use. Visit http://www.dovepress.com/testimonials.php to read real quotes from published authors.

Submit your manuscript here: http://www.dovepress.com/drug-design-development-and-therapy-journal 